\title{
The F-region trough: seasonal morphology and relation to interplanetary magnetic field
}

\author{
M. Voiculescu ${ }^{1}$, I. Virtanen ${ }^{2}$, and T. Nygrén ${ }^{2}$ \\ ${ }^{1}$ Department of Physics, Faculty of Sciences, University “Dunărea de Jos" Galati, St. Domnească, No. 47, 800008 Galati, \\ Romania \\ ${ }^{2}$ Department of Physical Sciences, University of Oulu, P.O. Box 3000, FIN-90014, Finland
}

Received: 7 July 2005 - Revised: 16 December 2005 - Accepted: 21 December 2005 - Published: 7 March 2006

\begin{abstract}
We present here the results of a statistical study of the ionospheric trough observed in 2003 by means of satellite tomography. We focus on the seasonal morphology of the trough occurrence and investigate the trough latitude, width and the horizontal gradients at the edges, at different magnetic local times, as well as their relations to geomagnetic activity and the interplanetary magnetic field. A seasonal effect is noticed in the diurnal variation of the trough latitude, indicating that summer clearly differs from the other seasons. In winter the troughs seem to follow the solar terminator. The width of the trough has a diurnal variation and it depends on the season, as well. The broadest troughs are observed in winter and the narrowest ones in summer. A discontinuity in the diurnal variation of the trough latitude is observed before noon. It is suggested that this is an indication of a difference between the generation mechanisms of morningside and eveningside troughs. The density gradients at the edges have a complex dependence on the latitude of the trough and on geomagnetic activity. The photoionization and the auroral precipitation are competing in the formation of the trough walls at different magnetic local times. An important finding is that the interplanetary magnetic field plays a role in the occurrence of the trough at different levels of geomagnetic activity. This is probably associated with the topology of the polar cap convection pattern, which depends on the directions of the IMF components $B_{y}$ and $B_{z}$.
\end{abstract}

Keywords. Ionosphere (Ionospheric disturbances; Auroral ionosphere)

\section{Introduction}

The ionospheric trough is a plasma density depletion observed at F-region heights at latitudes around $55^{\circ}-75^{\circ}$. It is elongated in longitudinal direction and its width in the latitudinal direction is of the order of $5-10^{\circ}$. Terms such as

Correspondence to: M. Voiculescu

(mirela.voiculescu@ugal.ro) main trough, mid-latitude trough, high-latitude trough, dayside trough and nightside trough are used to describe this phenomenon. Both ionosonde and radar data, and satellite beacon transmissions have been applied in studying the midlatitude trough for more than four decades (Moffet and Quegan, 1983), while studies of the high-latitude trough started later (Rodger et al., 1992). During the last decade, satellite tomography has become a useful tool for monitoring the ionosphere. This technique allows the observation of the Fregion within a wide latitudinal range and on a continuous basis, therefore it is very suitable for studies of the trough morphology (Jones et al., 1997; Kersley et al., 1997; Pryse et al., 1998).

The current knowledge of the phenomenology of the trough is relatively incomplete. The investigations have revealed several properties of the trough, but the understanding of these characteristics, for example, the formation and occurrence, is still at least partly unclear. The contradictions may origin from differences in the properties of the midlatitude and high-latitude troughs or the dayside and nightside troughs, as well as from a restricted time of observation. Some trough studies are based on limited time intervals (Whalen, 1989) or isolated cases (Jones et al., 1997; Pryse et al., 1998), while others make use of larger databases (Mallis and Essex, 1993; Kersley et al., 1997; Werner and Prölss, 1997; Horvath and Essex, 2003).

The main difference between the mid-latitude and highlatitude trough originates from the location of the trough relative to the auroral oval. According to Rodger et al. (1992), the former occurs equatorward of the auroral oval, while the latter is found inside or poleward of the oval. Initially, the mid-latitude trough was considered to be a night phenomenon (Moffet and Quegan, 1983; Aladjev et al., 2001; Vlasov and Kelley, 2003), but Whalen (1989) and Mallis and Essex (1993) observed that troughs also extend to the afternoon sector, displaying a high variability. In their review, Rodger et al. (1992) refer to the mid-latitude trough as a primarily night phenomenon, but point out that the highlatitude troughs could be seen at other times of the day. 
Previous studies have shown that the latitudinal position of the trough has a diurnal variation with its southernmost location at night, and the trough also moves towards lower latitudes with increasing magnetic activity (Moffet and Quegan, 1983; Whalen, 1989; Rodger et al., 1992; Kersley et al., 1997). Empirical models have been developed which describe the location of the trough minimum as a function of local time and magnetic activity (Werner and Prölss, 1997; Karpachev et al., 1996).

The seasonal variation of the trough behaviour is not yet very clear. Whalen (1989) and Aladjev et al. (2001) observe that troughs are seen mostly in winter, Moffet and Quegan (1983) conclude that in summer the occurrence is restricted only to midnight, while Mallis and Essex (1993) and Horvath and Essex (2003) see troughs regularly in other seasons, as well. These inconsistencies may be partly caused by the limited number of observations or by the restricted latitudinal range covered by the method of observation. Also, dividing the observations into mid-latitude and high-latitude troughs may hamper the comparison of different studies.

The explanation of these properties is still incomplete. Simultaneous effects of plasma convection, precipitation, neutral winds and heating are usually invoked to explain the depletion. Nightside troughs are assumed to form in the vicinity of the equatorward edge of the convection cell, where stagnation results from a balance between westward convection flow and eastward corotation (Whalen, 1989; Rodger et al., 1992; Pryse et al., 1998; Collis and Häggström, 1988). The dayside troughs form in regions where the dayside high density plasma is displaced by the sunward convective transport and replaced by low density plasma carried from the nightside (Whalen, 1989; Rodger et al., 1992; Pryse et al., 1998; Mallis and Essex, 1993). In some particular cases the poleward wall of the trough coincides with the equatorward boundary of precipitation (Jones et al., 1997; Horvath and Essex, 2003). Other possible mechanisms are the upwelling of the neutral atmosphere, which makes the ionosphere more molecular, and the frictional heating due to high ion velocities. Both of them result in increasing the ion loss rate and to erosion in plasma density, which points to the importance of thermospheric winds, electric fields and/or changes in the neutral composition (Crickmore et al., 1997; Vlasov and Kelley, 2003). In their excellent review Rodger et al. (1992) analyse all the processes by which plasma depletion could occur and conclude that ion convection is of paramount importance to trough generation.

This paper attempts to build a unified view of the morphology of the trough. We make no distinction between the different types of troughs and we agree with Whalen (1989), who suggests that the dayside and nightside troughs or the midlatitude and high-latitude troughs are manifestations of the same phenomenon. Our study aims to improve the present knowledge about the diurnal and seasonal variations of the trough for different geomagnetic levels. The width and the gradients at the edges are also included. In the present study we have chosen the edges as indicators of the trough position, in order to contribute to identifying the mechanism which contributes to the formation of the trough walls. The convection pattern, which according to many papers (Whalen, 1989; Rodger et al., 1992; Pryse et al., 1998) has a major effect on the trough formation, is governed by the orientation of the IMF. Therefore, the trough occurrence should be related to the IMF. Rodger et al. (1992) suggest that establishing the location of the trough during different IMF conditions would be of great benefit for clarifying the process of the trough formation. Although some comments on the IMF effect on the trough are presented by Rodger et al. (1992) and Pryse et al. (1998), there is no systematic study of this possible link and its implications for the formation of the trough. We investigate here, for the first time, the relationship between the IMF structure and the occurrence of the trough. Our conclusions are based on a study of the mid-latitude trough, using tomographic images of the high-latitude F-region, covering one year towards sunspot minimum (2003). We show in the following that some of our observations confirm previous findings, while others give new information on the trough behaviour and hopefully open new roads for the investigation of the processes which lead to the formation of the trough.

\section{Data analysis}

Tomographic images obtained from data collected by the Finnish tomography chain were used in the analysis. The chain is run by the Sodankylä Geophysical Observatory. The receivers of the chain are located at Troms $\varnothing\left(69.66^{\circ} \mathrm{N}, 18.94^{\circ} \mathrm{E}\right)$, Kilpisjärvi $\left(69.02^{\circ} \mathrm{N}\right.$, $\left.20.86^{\circ} \mathrm{E}\right)$, Kiruna $\left(67.84^{\circ} \mathrm{N}, 20.41^{\circ} \mathrm{E}\right)$, Luleå $\left(65.58^{\circ} \mathrm{N}\right.$, $\left.22.17^{\circ} \mathrm{E}\right)$ and Kokkola $\left(63.83^{\circ} \mathrm{N}, 23.06^{\circ} \mathrm{E}\right)$, and they observe the phase differences of the $150-\mathrm{MHz}$ and $400-\mathrm{MHz}$ signals transmitted by the Russian Low Earth Orbit (LEO) satellites. Electron density plots in the vertical plane above the receiver chain were produced by means of stochastic inversion (see, e.g. Nygrén et al., 1997). The latitudinal extent of the resulting tomographic images is about $15^{\circ}$.

As an example of the reconstructed ionosphere, an electron density plot showing a trough is given in Fig. 1. The top panel presents the tomographic result and, for comparison, a nearly simultaneous meridionally scanning incoherent scatter observation obtained by means of the EISCAT UHF radar is plotted in the bottom panel. The red dots indicate the positions of the satellite receivers and the radar. The curved structures in the bottom panel are artificially generated by the interpolation which was made in order to produce similar pictures for easier comparison.

The electron densities given by the two methods are not identical, which is due to the well-known fact that the tomographic measurements contain very little information on the vertical electron density profile. In this case the reconstructed layer is too compressed, which leads to a somewhat exaggerated maximum electron density. The tomographic result displays a bottomside bulge at the equatorward edge of the trough. This is caused by an enhanced E-layer, visible in the radar observation. The distance between the receivers is 


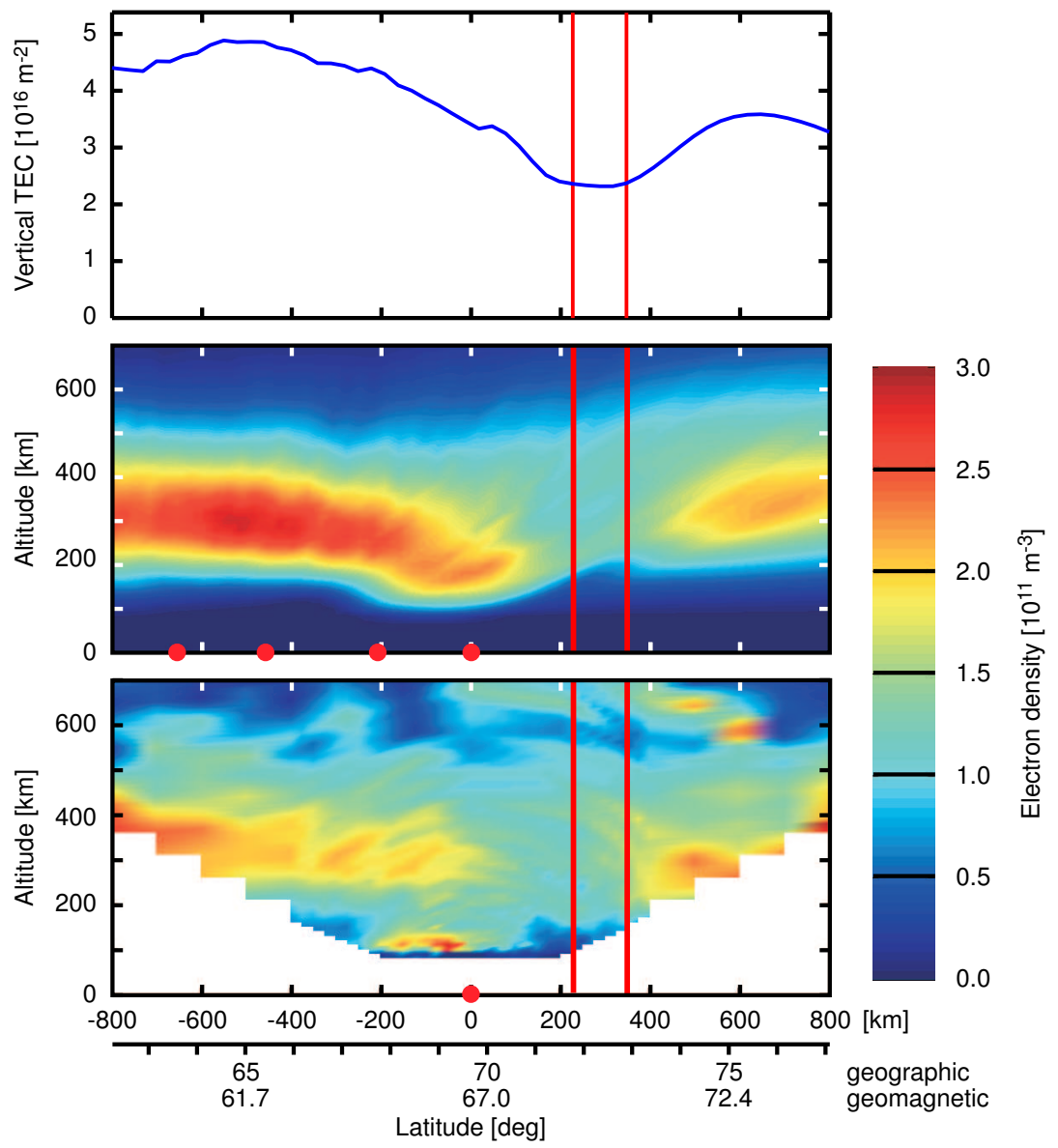

Fig. 1. Example of a trough observation by means of satellite tomography (middle panel) and the EISCAT UHF incoherent scatter radar (bottom panel). The top panel shows the vertical TEC between $200 \mathrm{~km}$ and $400 \mathrm{~km}$; the edges are marked with lines. The observations are from 25 June 2003 at about 01:00 UT. The red dots indicate the positions of the satellite receivers and the EISCAT UHF radar.

too long to allow a proper reconstruction of the E-region and, as a consequence, the E-region ionisation will show at higher altitudes. Still, the comparison reveals a trough nearly in the same position in both observations, even in this case when the trough is located somewhat outside of the receiver chain.

A search of troughs was made from all the data obtained in 2003. Cases with satellite passes too far from the receiver chain were first rejected. This led to a decrease of about $20 \%$ of the initial number of images. Next, an automatic search was made from the remaining set of observations. The latitudinal profile of average electron density between the heights of 200 and $400 \mathrm{~km}$ was calculated and troughs were identified from minima in these curves. The edges of the trough were defined as points where the height-integrated density drops to $50 \%$ from the outside value. This definition also puts a criterion to the definition of the trough; if the average electron density at a minimum is too great, no edge can be defined and the observation is not counted as a trough. There are cases when only one of the edges, either the poleward or the equatorward one, can be determined, but an observation is counted as a trough only if a minimum is visible within the field of view and the minimum is deep enough to allow the determination of at least one edge with the $50 \%$ criterion. The steepness of the trough edge was determined by calculating the derivative of the average electron density curve at the edge latitude. This result is the average value of the horizontal gradients of electron density within the $200-400-\mathrm{km}$ altitude range.

A visual check of all tomographic images was performed, and it confirmed that more than $95 \%$ of the cases were correctly identified as troughs. In a few cases the automatic search had interpreted some other features as troughs and these were removed from the data set. In spite of data gaps due to technical problems of the receivers, the resulting data set consists of more than 700 tomographic images with a trough. This number is large enough to allow a statistical study.

In conclusion, the data processing gives the geographic, invariant and corrected geomagnetic latitudes of the edges of the trough, the horizontal density gradients at the edges and the magnetic local time of observation. The width of the trough was calculated as the difference in the edge latitudes, only in cases when both edges have been determined. In addition, the values of the $K_{p}$ index were collected for the 

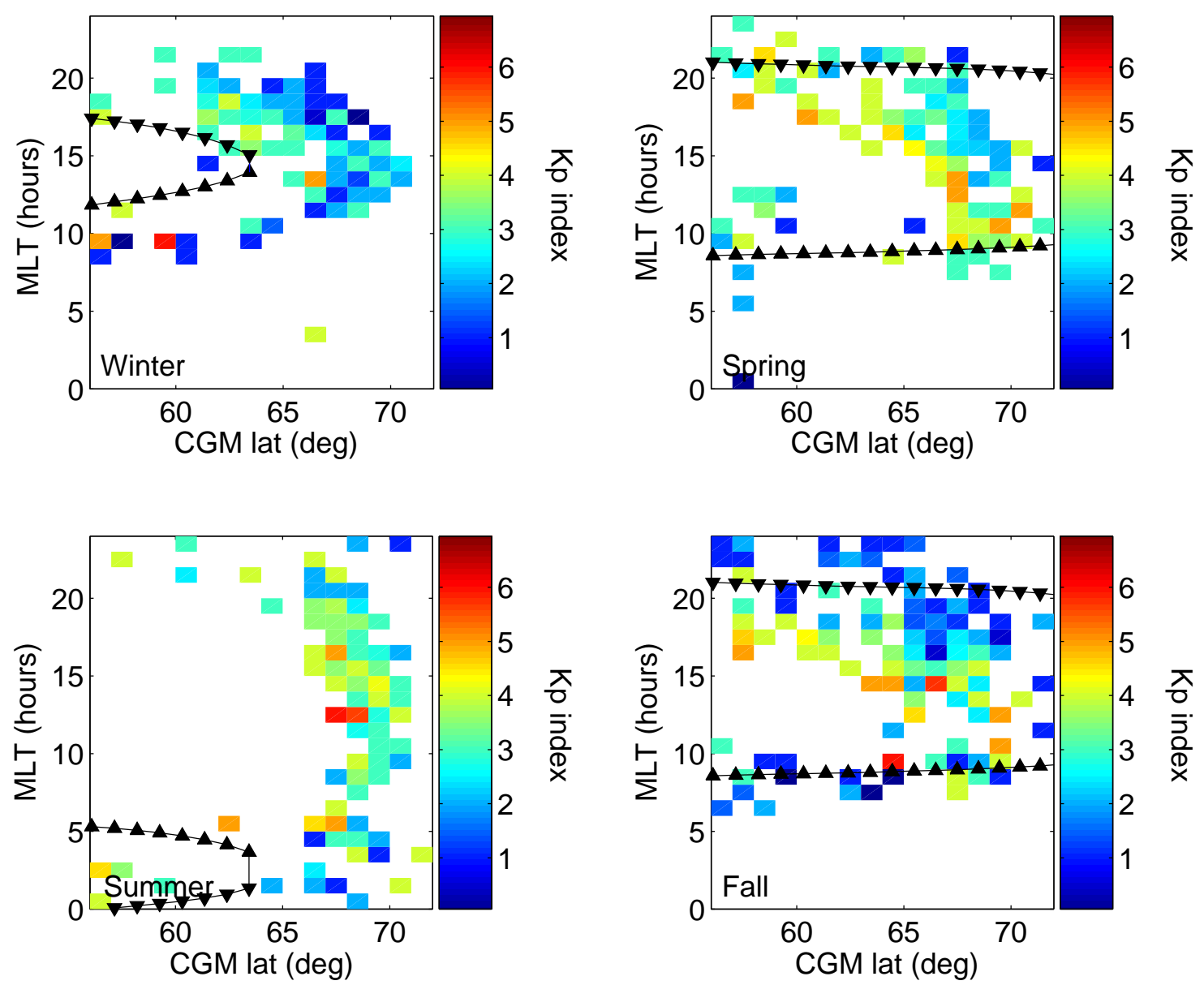

Fig. 2. The corrected geomagnetic latitudes and magnetic local times of the equatorward edges of the troughs observed in 2003 . The bin width is $1^{\circ}$ in latitude and $1 \mathrm{~h}$ in local time. The colour code indicates the mean $K_{p}$ during observations included in each bin. Also shown is the solar terminator at equinoxes and solstices, with triangles pointing towards the dayside.

purpose of studying the properties of the trough at different levels of magnetic activity. The interplanetary magnetic field data were taken from the solar wind observations provided by the WIND (January-June) and ACE (July-December) satellites. The delay time from the satellite position to the subsolar magnetosphere was taken into account and 15-min averages of the field components were computed. Finally, the mean values of all parameters were calculated for time bins of one hour and latitude bins of $1^{\circ}$.

\section{Results and discussion}

3.1 Diurnal and seasonal variation. Relation to magnetic activity

The observed troughs were divided into four groups, in order to investigate the diurnal variation in different seasons. Four months were taken for summer and winter, which leaves two months for each of the equinoxes. Spring was taken to cover March and April, summer to cover May, June, July and August, fall to cover September and October, and win- ter to cover November, December, January and February. The seasons have different lengths because the solar illumination changes more rapidly around equinoxes than around solstices.

Figures 2 and 3 show, respectively, the magnetic diurnal variations of the corrected geomagnetic latitude of the equatorward and poleward edges of the trough for every season. The colour code indicates the mean $K_{p}$ during the times of the trough observations included in each bin. Hence, the figures show the average positions of the edges of the trough observed at different levels of magnetic activity, but not the occurrence frequency. This representation allows a more comprehensive observation of the diurnal variation of the trough edges, together with its dependence of the magnetic activity and season. The geomagnetic coordinates have been chosen instead of the geographic ones, in order to identify the importance of the convection pattern on the formation of the trough. Moreover, the longitudinal variation of the trough, which would be better seen in a geographical framework, cannot be analyzed here, since the satellite is observing in a meridional plane. The solar terminator at equinoxes and 

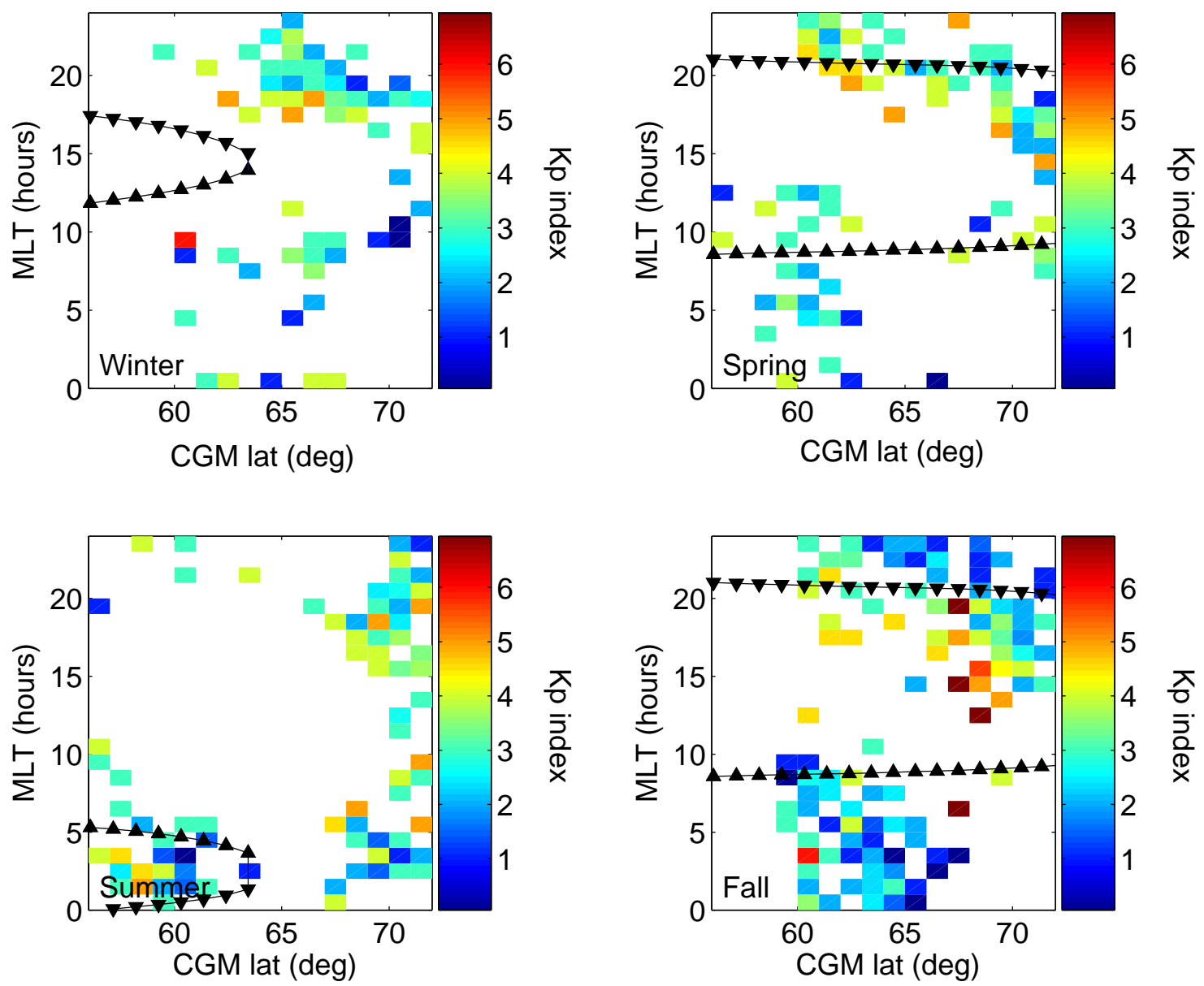

Fig. 3. Same as Fig. 2 for the observations of the poleward edges of the troughs observed in 2003.

solstices is also shown here, with triangles pointing towards the dayside.

The maximum latitude of both edges of the trough occurs around local geomagnetic noon or in the afternoon. In the afternoon and evening sector the trough moves towards lower latitudes in the course of local time. This is in agreement with previous findings (Moffet and Quegan, 1983; Whalen, 1989; Karpachev et al., 1996; Werner and Prölss, 1997; Kersley et al., 1997; Pryse et al., 1998). During the post-midnight and morning sectors the trough moves back to higher latitudes. In contrast to the post-noon sector, there is a remarkable difference in the trough behaviour between different seasons in the morning. This will be discussed in more detail below.

Figures 2 and 3 expose the well-known motion of the trough towards lower latitudes and/or the appearance of the trough at earlier local times when the geomagnetic activity level increases (Moffet and Quegan, 1983; Rodger et al., 1992; Pryse et al., 1998). This characteristic is more distinct for the equatorward edge of the trough and it appears in every season. In a few cases a trough is observed at high latitudes during an active geomagnetic period, but this is an exception rather than a rule. Rodger et al. (1992) have also found the same behaviour, indicating that the effect of mag- netic activity is more clearly seen at the motion of the equatorward edge. We made no attempt to compare our findings with model results of the trough location because these refer to the trough minimum while in the present paper only the edges are considered.

Since Figs. 2 and 3 show a regular diurnal behaviour of a latitudinal trough motion, it is difficult to make a distinction between high-latitude and mid-latitude troughs or between dayside and nightside troughs. In our observations the trough edges are seen within a latitudinal range of $56^{\circ}-72^{\circ}$ (corrected geomagnetic latitude) at all times of day. Therefore, we support the idea by Whalen (1989) that the mid-latitude and high-latitude troughs describe practically the same phenomenon observed at different latitudes at different times. Our observations cover a limited area between 60 and 75 geographical latitudes, thus troughs forming at higher latitudes (i.e. polar holes for instance) could not be observed.

In their review study Moffet and Quegan (1983) state that the trough is observed at 16:00-04:00 LT. Also, Vlasov and Kelley (2003) and Rodger et al. (1992) considered that the subauroral trough is mostly a phenomenon taking place in the nonilluminated ionosphere. A reason for observing troughs mostly in the nighttime may be a lack of high-latitude 
observations (Whalen, 1989). These results are, to some extent, in disagreement with our observations, which also reveal troughs in the daytime and in the morning, in all seasons except for winter, when the troughs occur overwhelmingly in darkness. At this time of the year, the equatorward edge follows closely the solar terminator. This shows that, at least in winter, the equatorward wall of the trough seems to coincide with the border between the solar illuminated high density F-region and the decayed plasma, which is in darkness. In the other seasons the trough occurs during both day and night. In addition to our study, some other investigations also show that the trough is not a predominantly nocturnal phenomenon, but it often appears in the daytime (Pryse et al., 1998; Horvath and Essex, 2003; Werner and Prölss, 1997). A similar result was presented by Mallis and Essex (1993), indicating that, in the Southern Hemisphere, the trough appears more often in the early afternoon and in the evening sector in all seasons, but also in the pre-dawn and morning sectors. Model results, on the other hand, predict the appearance of the trough in the evening/night/early morning sector (18:0006:00 MLT), extending only for UT=17:00 in the sunlight (Schunk, 1988).

Some evidence of the coincidence of the poleward trough edge with the equatorward edge of energetic particle precipitation has been given by Aladjev et al. (2001); Rodger et al. (1992) or Jones et al. (1997). As a consequence, the movement of the poleward edge of the trough towards lower latitudes with increasing geomagnetic activity may be explained by the expansion of the auroral oval in disturbed conditions. The explanation for the corresponding movement of the equatorward edge is less straightforward. Although, on average, the formation of troughs at lower latitudes and earlier in the evening is favoured by a higher $K_{p}$, this is not always true. This was also pointed out by Rodger et al. (1992) in their review paper, where they argued that $K_{p}$ is not the best indicator of the trough position.

The $K_{p}$ dependence is observed in winter, spring and autumn; summer is the exception. Both edges are observed mostly at high latitudes all day, regardless of the $K_{p}$ value. (The very few troughs observed at low latitudes are observed when the ionosphere is in darkness and they seem to be of a different origin. They will be discussed later.) This must be at least partly due to the solar illumination of the highlatitude F-region in summertime, which, of course, is independent of magnetic activity. In conclusion, Figs. 2 and 3 show clear differences between the trough behaviour in various seasons. This fact is evident especially in the variation of the equatorward edge. Spring and fall look rather similar. In winter the maximum latitudes of the southern edge are encountered at later magnetic local times in the evening than in other seasons. According to Mallis and Essex (1993) and Horvath and Essex (2003), the seasonal variations could be explained by the changing nature of the plasma composition and by the different solar influx and angle of incidence. The seasonal variation of the trough location is rather small in comparison with the diurnal one. Nevertheless, the seasonal effect is seen in the diurnal variation of the trough edges and in their relation to the magnetic activity. Interestingly, the daytime troughs seem to occur rarely if the magnetic activity is low. This is seen in both Figs. 2 and 3, especially during fall and winter. Model results of Schunk (1988) do not predict the dayside trough for given levels of precipitation and $K_{p}$. Our observations support the idea that these two factors have a major impact on the formation of the dayside trough due to their effect on the ion loss rate.

A closer look at Figs. 2 and 3, especially at the spring and fall plots, suggests that the troughs might be split into two categories. In the afternoon and pre-midnight sectors the northern edge is clearly at higher latitudes than in the postmidnight and morning sectors. On the morning side the equatorward edge may be even outside of the field of view. The postmidnight and morning troughs occur at low latitudes during periods of low geomagnetic activity, as well. A steep step in the trough position is visible somewhere close to noon.

The existence of the morning trough has been shown in other works, as well (Whalen, 1989; Horvath and Essex, 2003; Kersley et al., 1997; Pryse et al., 1998; Mallis and Essex, 1993). Especially in summer, the northward edge of the post-midnight and morning troughs lies either at high or low latitudes and, in between, there is a range of latitudes where the edge is only rarely observed. The observed step in the trough position is in line with Rodger et al. (1992), who argued that the different flow regimes should lead to a demarcation between duskside troughs and nightside/morning troughs. Such a discontinuity also appears in one of the models proposed by Werner and Prölss (1997) for the location of the trough minimum function of the magnetic local time. This shows that troughs at different times of day are caused by different combinations of physical processes.

The dependence of trough occurrence on magnetic activity was also investigated in each season. The number of trough observations when $K_{p}>4$ was compared with the total number observed. The resulting fractions are $45 \%$ for spring, $39 \%$ for summer, $25 \%$ for fall and $18 \%$ for winter. Hence, from September to February, the majority of the troughs (75\%-80\%) occur at relatively low levels of geomagnetic activity while for the rest of the year the $K_{p}$ index is high for almost half of the troughs. A possible explanation is that, in the latter case, shallow troughs are easily filled by plasma due to solar illumination and therefore troughs are not so often observed when geomagnetic activity is low. Our observations are not in line with Moffet and Quegan (1983), who found that the trough occurrence is greater for high magnetic activity. The basic reason for the disagreement could be the same as for the disagreement on diurnal occurrence, i.e. a limited latitude range of observations. Since troughs move towards lower latitudes with increasing magnetic activity, they also appear more often at low latitudes when magnetic activity is high. At low levels of magnetic activity the troughs are located at higher latitudes and thus may remain unobserved if the latitudinal range of observations is too limited. This may completely distort the conclusions to be made on the relation between occurrence and magnetic activity. 

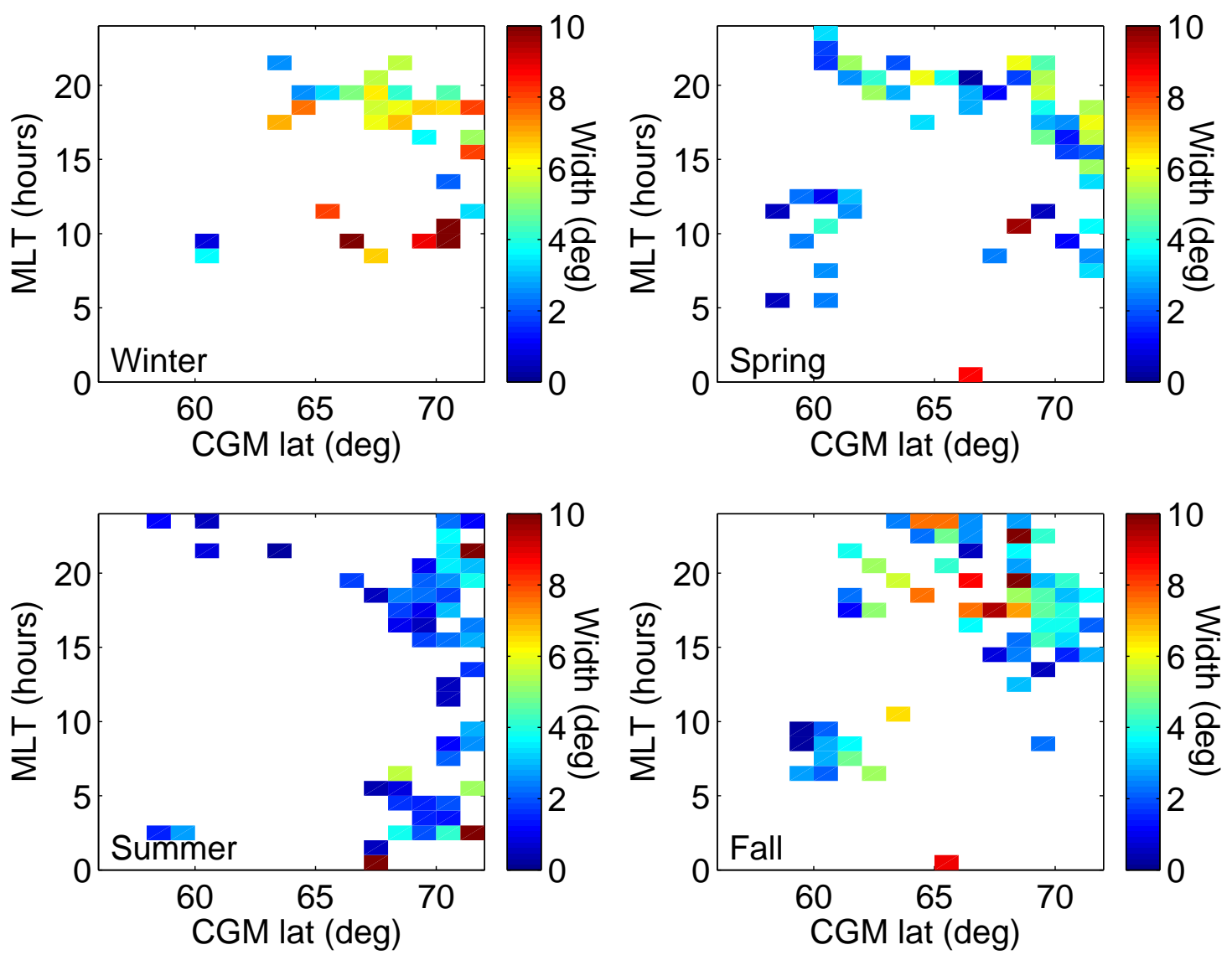

Fig. 4. The mean value of the trough width at different geomagnetic latitudes and magnetic local times of the poleward edge.

\subsection{Width}

Due to the fact that sometimes only one of the trough edges lies within the field of view of the tomography experiment, the trough width cannot be determined in all cases. Therefore, the statistics available for studying the width is smaller than that used in studying the positions of the edges.

Figure 4 displays the mean width at different latitudes and local times. The average width is shown in terms of the colour code and each rectangle indicates the average magnetic coordinates of the northern edge of the troughs used in calculating the respective average. The width ranges from 0.5 to $12^{\circ}$, which is a greater interval than the $5-9^{\circ}$ mentioned by Rodger et al. (1992). However, a comparison with other results may, however, be misleading, due to the different criteria used in calculating the width of the trough.

A clear seasonal variation of the trough width is seen in Fig. 4. The broadest troughs are observed in winter and the narrowest ones in summer, while the equinox seasons represent an intermediate case. In winter the width often reaches $10^{\circ}$ or more, but in summer the trough is only $0.5^{\circ}-3^{\circ}$ wide. The seasonal dependence is most likely explained by the fact that the F-region electron density is enhanced in the equatorward part of the trough region by the solar illumination in summer and reaches higher latitudes. The seasonal vari- ation of the trough width and position suggests that a thorough analysis of the trough formation, for particular cases, should consider the effect of both geographical and geomagnetic coordinates. Although the diurnal variation of the width is not very clear, there is some indication of narrow troughs around noon and broader ones in the afternoon and in the morning. This observation is not in good agreement with that by Mallis and Essex (1993), who see maximum widths at 09:00-15:00 LT.

\subsection{Wall gradients}

No significant seasonal variation was found in the gradients of the edges. Therefore, the diurnal dependence of the wall gradients from all seasons is presented in a single plot. The results from the two walls are shown in Fig. 5. The rectangles indicate the magnetic latitude and local time of the average trough edge, and the mean values of the gradients are shown in terms of the colour code. The gradients are negative at the equatorward edge and positive at the northern edge.

The diurnal variation of the gradients at the equatorward and poleward edges display a clearly different behaviour. At the equatorward edge the gradient is usually gentle from early morning up to the magnetic noon, but then its soon becomes steep in the afternoon sector. At the poleward edge the gradient is gentle most of the day and steep gradients are 

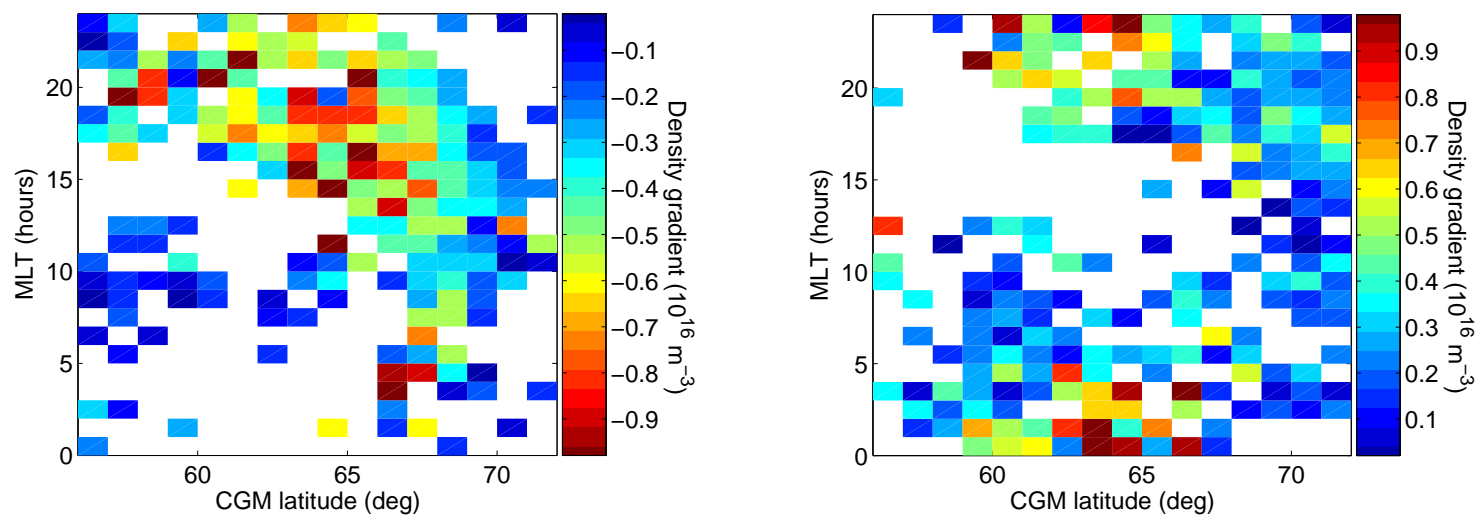

Fig. 5. The mean electron density gradient at the equatorward edge (left) and poleward edge (right) at different geomagnetic latitudes and magnetic local times. The rectangles indicate the location of the edge and the value of the gradient is shown in terms of colour code.
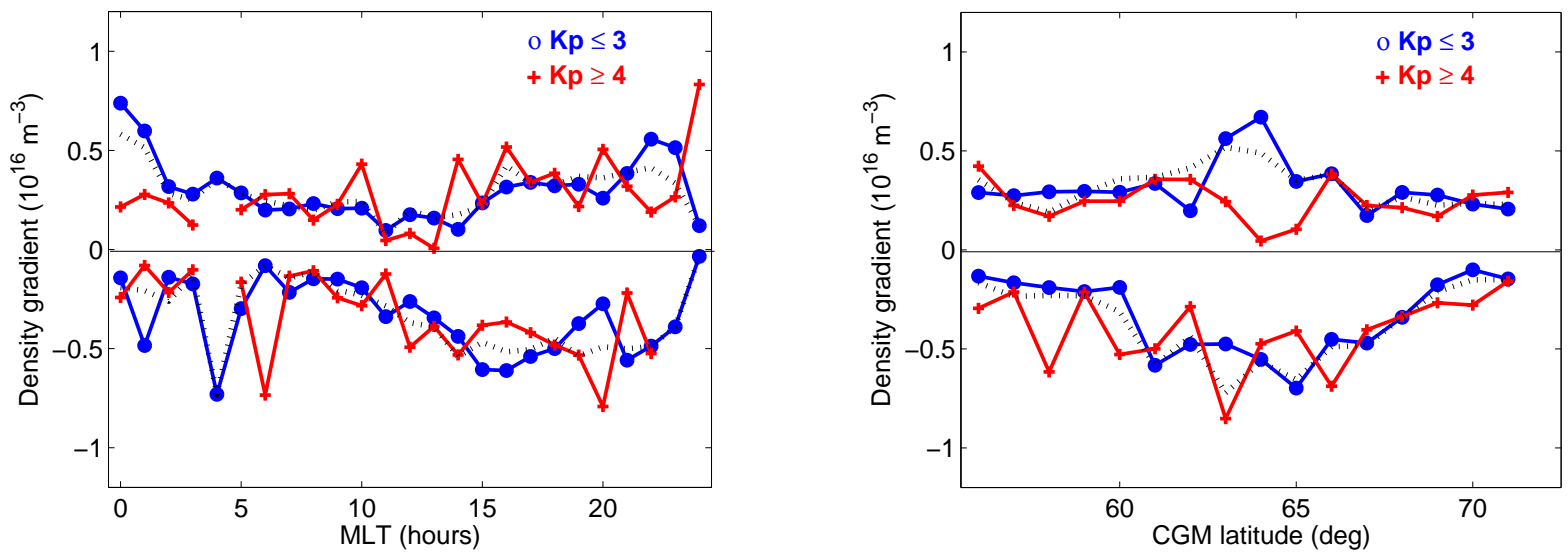

Fig. 6. The diurnal (left) and latitudinal (right) variation of the electron density gradient at the edges of the trough. Positive values refer to the poleward and negative values to the equatorward edge. The heavy dotted lines indicate the mean value of all observations, and the red and blue ones the mean values from times of $K_{p}>4$ and $K_{p}<3$, respectively.

observed only around midnight. In other words, the poleward wall is steeper when the F-region is not illuminated, while the gradient at the equatorward wall is higher when the photoionization maintains the plasma in the F-region. This gives a hint to the relative importance of the geographical (solar dependence) and geomagnetic (convection pattern and precipitation) effects in the formation mechanisms of the trough.

A different view of the latitudinal and diurnal behaviour of the gradients is shown in Fig. 6. Here the left-hand panel displays the mean diurnal variation and the right-hand panel the mean latitudinal variation of the trough gradients. The average of all observations is shown by the heavy dashed line, and averages calculated from times with $K_{p} \leq 3$ and $K_{p} \geq 4$ by blue and red lines, respectively. Error bars are not shown and only points within the limit of one standard deviation have been considered.

Figure 6 indicates that the poleward edge is steeper than the equatorward one at magnetic midnight. Then the poleward gradient decreases nearly continuously until it starts increasing at noon. The gradient at the southward edge keeps more or less constant between midnight and about 09:00 MLT (assuming that the observations at 04:00 and 06:00 MLT are outliers). After this, its absolute value starts to increase, obtaining a maximum in the evening sector. The result of this behaviour is that, from magnetic midnight up to about 08:00-10:00 MLT, the poleward edge is steeper than the equatorward one. Then the equatorward edge becomes steeper than the poleward one and this continues up to the magnetic midnight. Hence, most of the day, the steepest edge is encountered at the equatorward edge of the trough.

Jones et al. (1997) found that the gradients increase with time in the post-noon sector. This is in agreement with our results. Jones et al. (1997) and Kersley et al. (1997) also found that, during quiet magnetic periods, the poleward edge is steeper than the equatorward one. Similarly, Rodger et al. (1992) refer to a higher poleward gradient, especially in the pre-midnight sector. This seems to be at least partly in contradiction with our observations, showing that most of the time the equatorward edge is the steeper one. On the other hand, within a few hours around magnetic midnight 

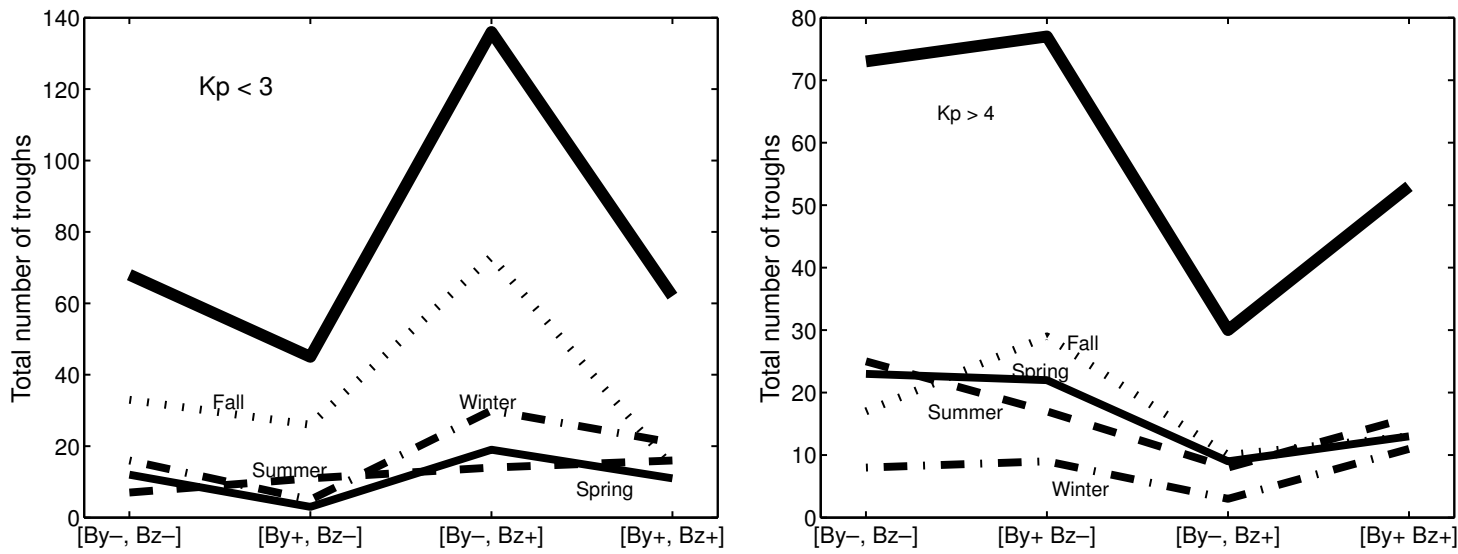

Fig. 7. The number of observed troughs during low (left) and high (right) magnetic activity when the IMF points into each of the four quadrants in the $B_{y} B_{z}$ plane. The numbers are also shown for each season.

the poleward edge is clearly steeper, but only during low geomagnetic activity. Kersley et al. (1997) report a steepening of the walls with increasing $K_{p}$ in the pre-midnight sector. No such dependence is visible in Fig. 6. Our results indicate that magnetic activity has no clear effect on the gradients, except around magnetic midnight, and the diurnal variation of the equatorward gradient does not show any clear correlation with the $K_{p}$ index.

A clear latitudinal dependence of the gradient at both edges of the trough is seen in the right-hand panel of Fig. 6. On average, the edges are at their steepest around $63^{\circ}-65^{\circ}$ in magnetic latitude. The maximum of the equatorward gradient is somewhat higher than that of the poleward gradient. The opposite situation is found both at high and low latitudes; there the northward edge is clearly steeper than the southward edge occurring at the same latitudes. A comparison with Fig. 5 shows that these troughs come from all times of the day, while the high gradients come from the afternoon or midnight sectors. The main effect of magnetic activity is seen at the poleward edge. When the magnetic activity is high, the curve shows a minimum at the very same latitudes where the total curve and the curve at low magnetic activity have a maximum. This decrease could be the effect of particle precipitation associated with the expansion of the auroral oval at higher $K_{p}$, which could also fill in the troughs. At the equatorward edge, there is some indication of the gradient becoming steeper at low latitudes when $K_{p}$ increases. This is in agreement with Rodger et al. (1992) and Horvath and Essex (2003), who noticed that a storm leads to an enhancement of TEC equatorward of the trough. Also, Schunk (1988) shows that an upward plasma drift leads to an increased density equatorward of the trough. No similar variation is seen at the poleward wall at the low-latitude part of the curve.

\subsection{Relation to the interplanetary magnetic field}

The effect of the interplanetary medium on the occurrence of the trough was investigated using the IMF components $B_{z}$ and $B_{y}$. Out of these, the $B_{z}$ component has the most important effect on the magnetosphere-ionosphere coupling, and therefore it should have the greatest effect on the trough, as well. Using the IMF data, the troughs were divided into four categories, according to the prevailing signs of $B_{y}$ and $B_{z}$, during each observation. Troughs during low and medium/high geomagnetic activity were studied separately.

Figure 7 shows the distribution of the total number of observed troughs for the four possible combinations, for the signs of $B_{y}$ and $B_{z}$ during low (left) and medium/high magnetic activity (right). Distributions for each season are also given. During low geomagnetic activity, the number of observed troughs is greatest when $B_{z}$ is positive and $B_{y}$ is negative. In the other three cases the numbers are nearly similar, although a minimum is obtained when $B_{y}$ is positive and $B_{z}$ negative. This indicates that both IMF components have their effect on the trough formation. When geomagnetic activity is increased, $B_{z}$ has a more dominant role. Regardless of the sign of $B_{y}$, the number of troughs is higher when $B_{z}$ is negative. For positive $B_{z}$, a dependence on the sign of $B_{y}$ can be again noticed; more troughs are then observed when $B_{y}$ is positive. The distributions follow the same pattern during all seasons except summer.

In Fig. 8 the sign of the average $B_{z}$ during observed troughs is presented in terms of colour code; positive is shown by red and negative by blue. Each rectangle indicates the geomagnetic latitude and local time of the poleward/equatorward edge of a trough, and the signs are calculated from the values of $B_{z}$ associated with the troughs belonging to each rectangle. Separate plots are made for poleward and equatorward edges, as well as for low and medium/high $K_{p}$ values. Figure 9 is a similar presentation for $B_{y}$. 

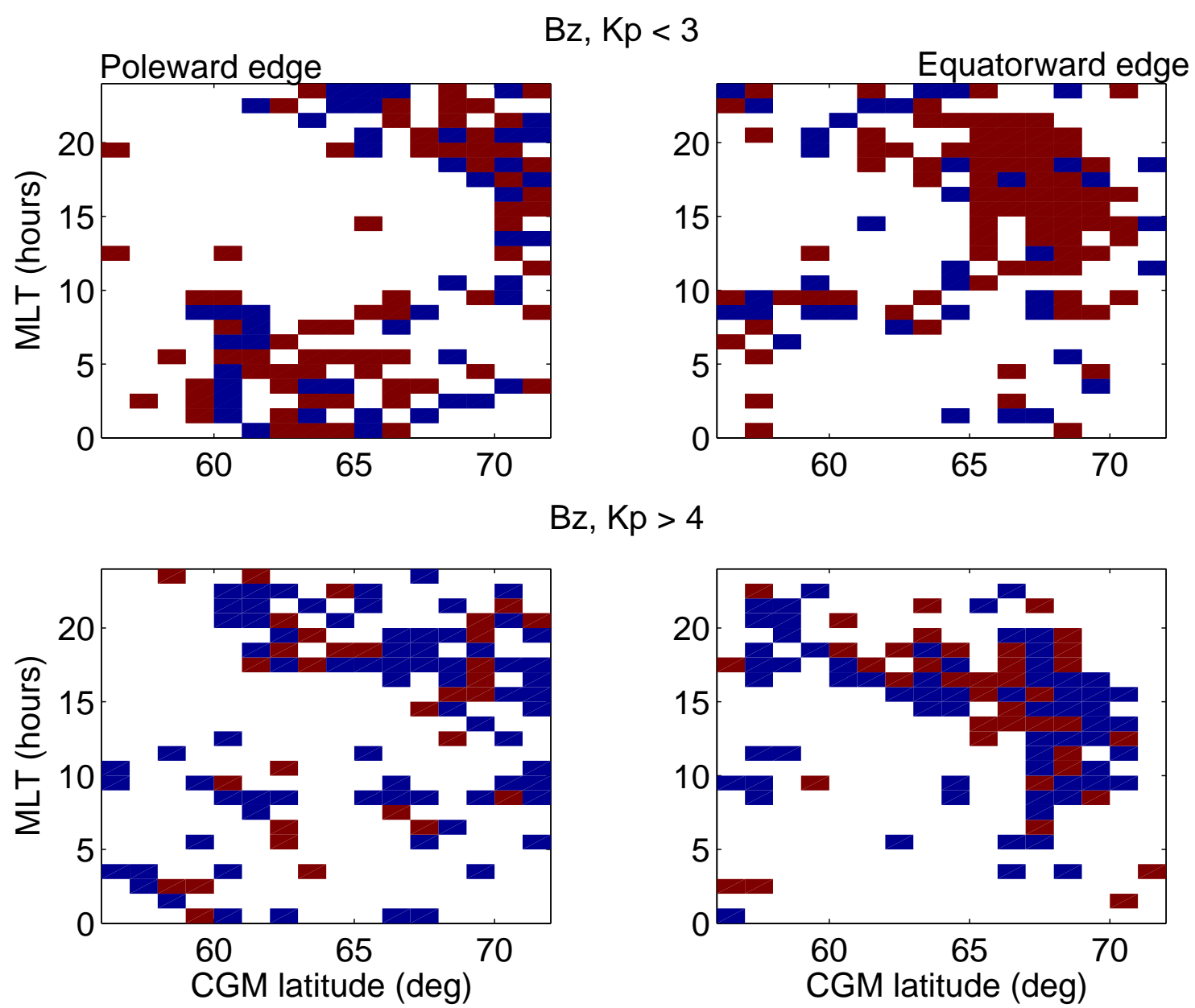

Fig. 8. The average sign (red means positive and blue negative) of $B_{z}$ during observations of trough edges at different magnetic latitudes and local times. Poleward and equatorward edges are shown in the left and right panels, respectively. Top panels contain troughs observed when $K_{p}<3$ and bottom panels when $K_{p}>4$.

Both top panels in Fig. 8 show more red than blue, while blue is dominant in the bottom panels. This indicates that $B_{z}$ is mostly positive when troughs are observed during periods of low magnetic activity. When magnetic activity is higher, negative $B_{z}$ favours the occurrence of a trough. These observations could be partly explained by the fact that the level of geomagnetic activity is controlled by the $z$ component of the IMF. However, high geomagnetic activity levels have also been observed together with positive, large or highly variable, $B_{z}$. For both low and high geomagnetic activity the equatorward edge position seems to be clearly defined by the orientation of $B_{z}$. For small $K_{p}$, the majority of the noon and evening troughs coincide with positive $B_{z}$. A negative $B_{z}$ is generally associated with troughs whose equatorward edge is at lower latitudes, occurring at night or in the morning. When $K_{p}$ is high, the troughs are dominated by negative $B_{z}$. There is also some indication that, when $B_{z}$ is negative, the trough is located at lower latitudes than in the opposite case. These panels also reveal the demarcation between troughs occurring in the post-noon sector and those forming at night or in the morning. The latter type of troughs form mostly when geomagnetic activity is low. If $K_{p}$ is higher, these troughs are clearly associated with negative $B_{z}$. The fact that the role of $B_{z}$ is better seen in the equatorward edge than in the poleward one sustains the hypothesis that the equatorward boundary is correlated with the equatorward threshold of the ionospheric effects of convection (Whalen, 1989).

In Fig. 9 blue is dominant in the top panels and red in the bottom panels. Hence, $B_{y}$ also controls the occurrence of the trough. At low levels of magnetic activity, negative values of $B_{y}$ are favouring the trough, but when the magnetic activity increases, the occurrence of troughs is favoured by positive $B_{y}$. Unexpectedly, for quiet times $B_{y}$ seems to play a more important role in the position of the troughs than $B_{z}$. The appearance of a trough at noon when $K_{p}$ is low is more probable if $B_{y}$ is positive. This is also valid for higher geomagnetic activity levels, when, however, a positive $B_{y}$ seems to generally favor the occurrence of troughs.

Tentatively, some attempt can be made to explain the observations in Figs. 7-9. We assume that the northward edge 


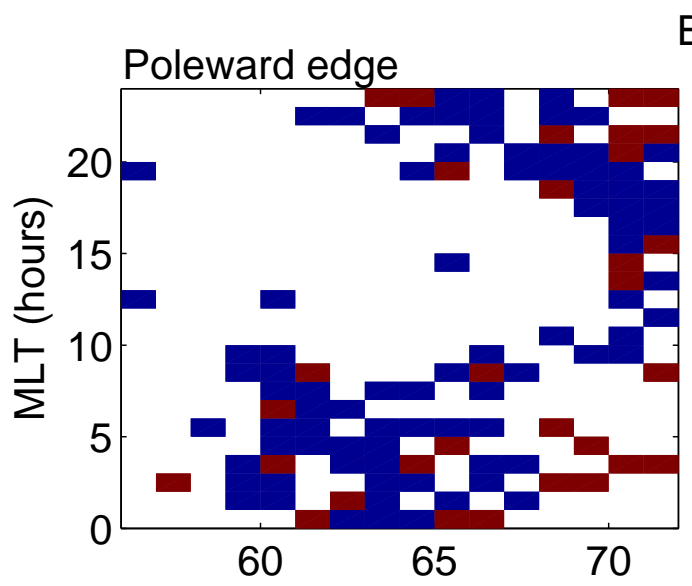

By, $\mathrm{Kp}<3$

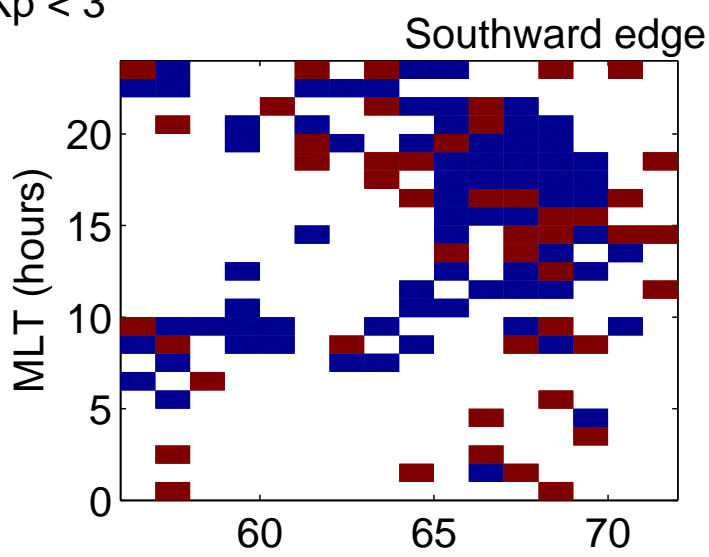

By, $\mathrm{Kp}>4$
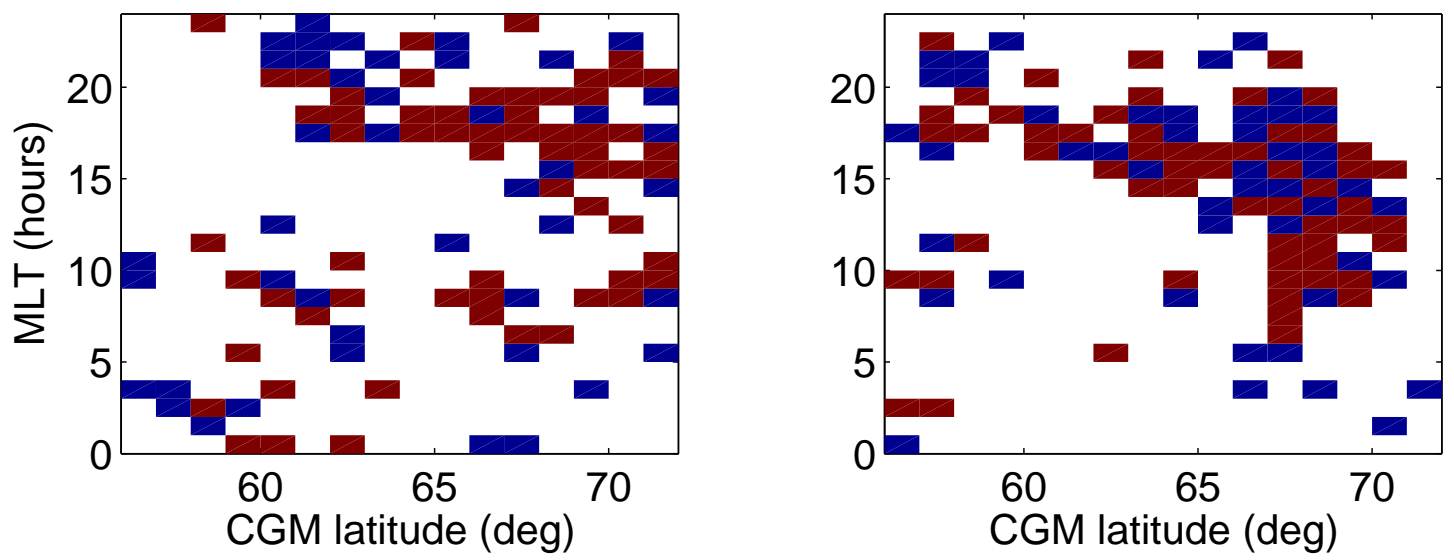

Fig. 9. Same as Fig. 8 for $B_{y}$.

of the trough is often associated with precipitation and the equatorward edge lies at the equatorward border of the convection pattern. The dayside trough forms in the vicinity of the return flow of the dusk convection cell (Pryse et al., 1998) in regions where the sunward convective transport displaces the high density plasma with the low density plasma from the nightside (Whalen, 1989). The nightside trough is created within the stagnation region which appears where the westward convection and eastward corotation more or less cancel each other. Besides the decay of the plasma concentration, low density plasma is transported by the westward convection from later night local times. The size and structure of the convection pattern is controlled by the IMF. When $B_{z}$ is positive, the extent of the convection pattern is limited by the small polar cap. When $B_{z}$ is negative, the polar cap and the convection pattern extends to lower latitudes. The latitudes of particle precipitation are largely but not absolutely connected with the convection pattern. If $B_{z}$ is positive and $K_{p}$ is low, precipitation region may lie to the north of the narrow stagnation region, making the northern edge of the trough. If the stagnation region remains narrow and the precipitation region moves equatorwards, plasma production by precipita- tion may compensate the long-time loss within the stagnation region and no trough will appear. These two cases might explain the fact that, in Fig. 8, troughs during positive $B_{z}$ tend to disappear when $K_{p}$ increases.

When $B_{z}$ is negative, the stagnation region is wider and extends towards lower latitudes. Weak magnetic activity means weak precipitation and a precipitation region located probably at high latitudes. Then it is possible that the density depletion is so shallow that it will not meet our criteria of a trough search and it is not counted as a trough. Precipitation is intensified at high values of $K_{p}$ and it may also move equatorwards. Then a more pronounced poleward edge is formed and the depletion will be counted as a trough. This could contribute to the fact that, during negative $B_{z}$, troughs are observed at high values of $K_{p}$.

The role of $B_{y}$ in the generation of the trough may be associated with the basic difference in the convection patterns for different signs of $B_{z}$. When $B_{z}$ is negative, the convection pattern consists of two cells and the main effect of $B_{y}$ is to change the relative sizes of the dawn and dusk cells. Figures 8 and 9 show that during high geomagnetic level the troughs are favoured by negative $B_{z}$ and positive 
$B_{y}$, when the plasma transport velocity is enhanced. When $B_{z}$ is positive the sign of $B_{y}$ determines the flow direction in the innermost cells and therefore has a major effect on the plasma flow. When $K_{p}$ is low, Fig. 7 indicates that, for positive $B_{z}$, troughs are less probable when $B_{y}$ is positive. The convection pattern has three or four small cells. Models of high-latitude convection patterns (Ruohoniemi and Greenwald, 1996) show that in this case the plasma stagnates for a short time, between about 19:00 and 21:00 MLT, at latitudes which are too high to be observed by the tomographic chain. In the nightside, corotation can no more be cancelled by the weaker convection flow. This prevents plasma stagnation and may hinder the formation of the trough. Moreover, we suggest that, in this case, the flux of low-density plasma from the nightside is reduced and therefore the formation of the dayside trough becomes less probable.

The above considerations should only be regarded as a general discussion of the complicated process of trough generation. Extensive model calculations would be needed for a true explanation of all observations.

\section{Conclusions}

This paper confirms the well-known characteristics of the trough behavior. The troughs appear throughout the day and move towards lower latitudes with increasing local time during the afternoon and evening hours. Secondly, the trough also moves to lower latitudes and appears earlier in the afternoon, when magnetic activity increases. In our data these results are better seen for the equatorward edge of the trough.

Our results also present new findings on the occurrence of the troughs. The fact that their latitude changes in a continuous manner, from noon to evening and midnight, suggests that the troughs at these times are the result of the same processes, although the weight of different aspects of these processes may vary with local time. Interesting findings are associated with the morning-side troughs and with the discontinuity in the diurnal variation the trough latitude, observed before noon. When troughs occur at low latitudes in the morning, the geomagnetic activity is often relatively low and high values of $K_{p}$ are encountered only in a few cases. This behaviour differs from what is observed in the afternoon and evening sectors. Hence, morning troughs clearly differ from troughs observed in the evening, which gives a hint that there is also a difference in the generation mechanism. This is not surprising since, in the case of the double cell convection pattern, for instance, convection in the morning sector is in the same direction as corotation. Thus, stagnation is not expected in the same manner as before midnight. The occurrence of the morning troughs at low latitudes during low magnetic activity suggests that the formation of this particular type of trough cannot rely on processes induced by the convection.

Some seasonal effects on the occurrence of the trough have been found. In winter the maximum latitude is encountered at later local times than during other seasons. Most of the troughs occur in darkness and follow the solar terminator. This is seen especially in the equatorward edge, indicating that the photoionization plays an important role in the formation of the trough. Otherwise, troughs behave in a fairly similar way during winter and equinoxes. Summer is the most peculiar season; troughs are encountered mostly at high latitudes, even for high geomagnetic activity. The width varies with the season and is smaller in summer and greater in winter. Also, the trough width seems to attain a minimum around noon. A likely explanation for this observation is solar illumination extending to high latitudes in summer.

Another finding in this paper is that the orientation of IMF in the $y z$ plane controls the occurrence of the trough. It is suggested that this is connected to the topology of the polar cap convection pattern during different sign combinations of $B_{y}$ and $B_{z}$. The control is different at low and high levels of magnetic activity. This probably happens because magnetic activity is related to the size of the polar cap, thus to the orientation of $B_{z}$. Besides the anticipated dependence of the trough behaviour with the $z$ component, we found that the $y$ component also plays an important role. We suggest that this highlights the convection pattern role in the trough formation. Also, when magnetic activity is high, particle precipitation is more irregular and is encountered at a wider latitude region than at low activity levels. The location of the precipitation region with respect to the convection pattern is expected to have an effect on the occurrence of the trough.

The tomographic method gives a means of observing the F-region electron density within a wide latitudinal region. This has allowed us to obtain trough observations which show the whole diurnal variation of trough occurrence. A continuous registration throughout the year has also provided data from all seasons and geomagnetic as well as IMF conditions. The availability of satellites is the main limitation of the method. More data are expected to be available in the future, since the tomographic chain is operational. Finally, it should be emphasized that a trough is a complex phenomenon. Although some suggestions for explaining the observations are made in this paper, decisive explanations can only be obtained by means of sophisticated model calculations.

Acknowledgements. The Scandinavian tomography chain is run by Sodankylä Geophysical Observatory, Finland. We are grateful to T. Raita and J. Manninen for their continuous effort in maintaining the receiver chain and to M. Lehtinen, M. Markkanen and late J. Pirttilä for their efforts in developing the tomographic inversion routine. Grant (project No 51465) from the Academy of Finland and support from Space Institute at the University of Oulu is also gratefully acknowledged. EISCAT is an International Association supported by Finland (SA), France (CNRS), the Federal Republic of Germany (MPG), Japan (NIPR), Norway (NFR), Sweden (NFR) and the United Kingdom (PPARC).

Topical Editor M. Pinnock thanks A. Rodger and another referee for their help in evaluating this paper. 


\section{References}

Aladjev, G. A., Evstafiev, O. V., Mingalev, V. S., Mingaleva, G. I., Tereschenko, E. D., and Khudukon, B. Z.: Interpretation of ionospheric F-region structures in the vicinity of ionization troughs observed by satellite radio tomography, Ann. Geophys., 19, 2536, 2001, SRef-ID: 1432-0576/ag/2001-19-25.

Collis, P. N. and Häggström I.: Plasma convection and auroral processes associated with the main ionospheric trough at high latitudes, J. Atmos. Terr. Phys., 50, 339-404, 1988.

Crickmore, R. I., Jenkins, B., and Bailey, G. J.: Variations in the altitude of the F2 peak associated with trough-formation processes, Ann. Geophys., 14, 628-626, 1997,

SRef-ID: 1432-0576/ag/1996-14-628.

Horvath, I. and Essex, E. A.: The equatorward-hemisphere midlattude day-time and night-time trough at low sunspot numbers, J. Atmos. Solar-Terr. Phys., 65, 917-940, 2003.

Jones, D. G., Walker, I. K., and Kersley, L.: Structure of the poleward wall of the trough and the inclination of the geomagnetic field above the EISCAT radar, Ann. Geophys., 15, 740-746, 1997, SRef-ID: 1432-0576/ag/1997-15-740.

Karpachev, A. T., Deminov, M. G., and Afonin, V. V.: Model of the mid-latitude ionospheric trough on the base of Cosmos-900 and Intercosms-19 satellites data, Adv. Space Res. 18, 6, (6)221(6)230, 1996.

Kersley, L., Pryse, S. E., Walker, I. K., Heaton, J. A. T., Mitchell, C. N., Williams, M. J., and Willson, C. A.: Imaging of electron density troughs by tomographic techniques, Radio Sci., 32, 4, 1607-1621, 1997.

Mallis, M. and Essex, E. A.: Diurnal and seasonal variability of the equatorward hemisphere main ionospheric trough from differential-phase measurements, J. Atmos. Terr. Phys., 55, 7, 1021-1037, 1993.
Moffet, R. J. and Quegan, S.: The mid-latitude trough in the electron concentration of the ionospheric F-layer: a review of observations and modelling, J. Atmos. Terr. Phys., 45, 5, 315-343, 1983.

Nygrén, T., Markkanen, M., Lehtinen, M., Tereshchenko, E. D., and Khudukon, B. Z.: Stochastic inversion in ionospheric radiotomography, Radio Sci., 32, 2359-2372, 1997.

Pryse, S. E., Kersley, L., Williams, M. J., and Walker, I. K.: The spatial structure of the dayside ionospheric trough, Ann. Geophys., 16, 1169-1179, 1998, SRef-ID: 1432-0576/ag/1998-16-1169.

Rodger, A. S., Moffet, R. J., and Quegan, S.: The role of ion drfit in the formation of ionization troughs in the mid- and high-latitude ionsophere - a review, J. Atmos. Terr. Phys., 54, 1, 1-30, 1992.

Ruohoniemi, J. M. and Greenwald, R. A.: Statistical patterms of high-latitude convection obtained from Goose Bay HF radar observations, J. Geophys. Res., 101, A10, 21 743-21 763, 1996.

Schunk, R. W.: A mathematical model of the middle and high latitude ionosphere, Pageoph, 127, 2/3, 255-303, 1988.

Vlasov, M. N. and Kelley, M. C.: Modeling of the electron density depletion in the storm-time trough on April 20, 1985, J. Atmos. Solar-Terr. Phys., 65, 211-217, 2003.

Whalen, J. A.: The daytime F layer trough and its relation to ionospheric-magnetospheric convection, J. Geophys. Res., 94 A12, 17 169-17 184, 1989.

Werner, S. and Prölss, G. W.: The position of the ionospheric trough as a function of local time and magnetic activity, Adv. Space. Res., 20, 9, 1717-1722, 1997. 